\title{
Aircraft System Noise Prediction Uncertainty Quantification for a Hybrid Wing Body Subsonic Transport Concept
}

\author{
Jason C. June* and Russell H. Thomas ${ }^{\dagger}$ \\ NASA Langley Research Center, Hampton, VA, 23681, USA \\ Yueping Guo \\ NEAT Consulting, Seal Beach, CA, 90740, USA
}

\begin{abstract}
Aircraft system level noise prediction for advanced, unconventional concepts has undergone significant improvement over the past two decades. The prediction modeling uncertainty must be quantified so that potential benefits of unconventional configurations, which are outside of the range of empirical models, can be reliably assessed. This paper builds on previous work in an effort to improve estimates of element prediction uncertainties where the prediction methodology has been improved, or new experimental validation data are available, to provide an estimate of the system level uncertainty in the prediction process. In general, the uncertainty of the prediction will be strongly dependent on the aircraft configuration as well as which technologies are integrated. While the quantitative uncertainty values contained here are specific to the hybrid wing body design presented, the underlying process is the same regardless of configuration. A refined process for determining the uncertainty for each element of the noise prediction is detailed in this paper. The system level uncertainty in the prediction of the aircraft noise is determined at the three certification points, using a Monte Carlo method. Comparisons with previous work show a reduction of $1 \mathrm{EPNdB}$ in the $95 \%$ coverage interval of the cumulative noise level. The largest impediment for continued reduction in uncertainty for the hybrid wing body concept is the need for improved modeling and validation experiments for fan noise, propulsion airframe aeroacoustic effects, and the Krueger flap, which comprise the bulk of the uncertainty in the cumulative certification noise level.
\end{abstract}

\section{Nomenclature}

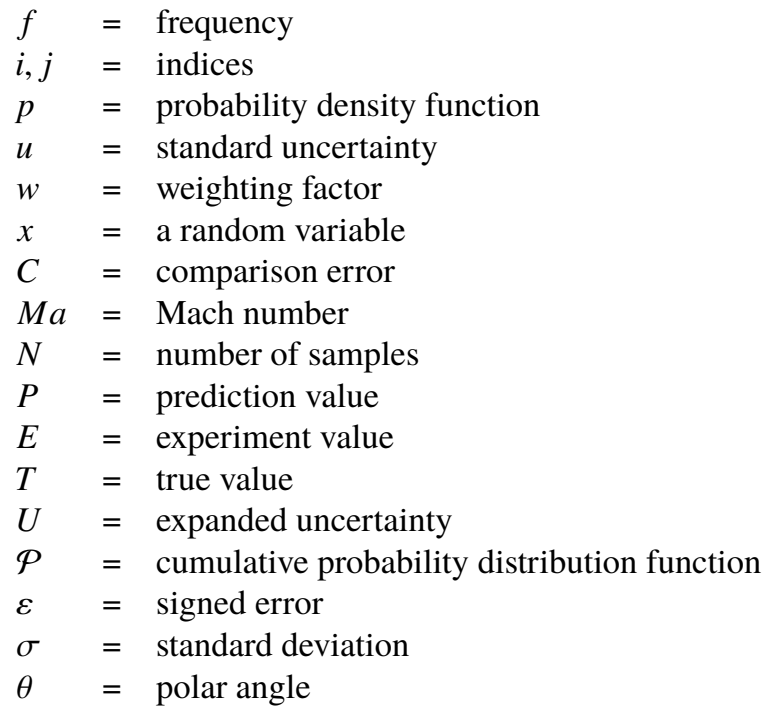

\footnotetext{
*Research Aerospace Engineer, Aeroacoustics Branch, MS 461, AIAA Member.

${ }^{\dagger}$ Senior Research Engineer, Aeroacoustics Branch, MS 461, AIAA Associate Fellow

†EAT Consulting, 3830 Daisy Circle, Seal Beach, CA 90740, AIAA Associate Fellow
} 


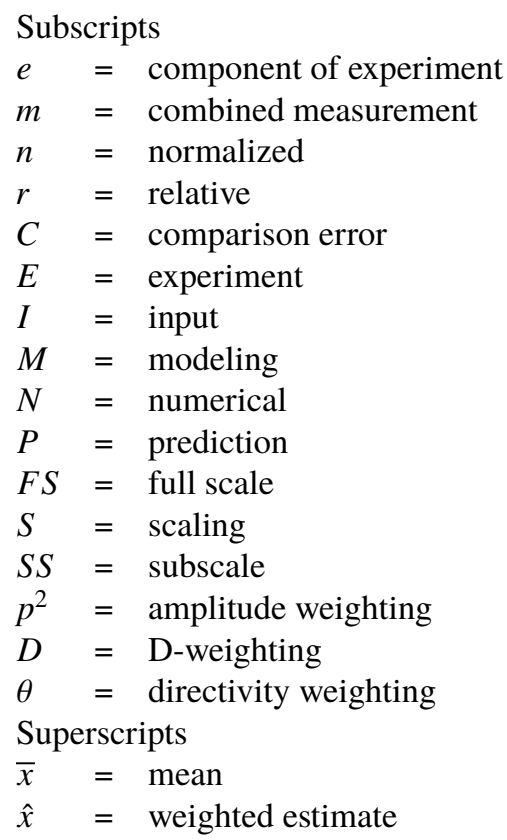

\section{Introduction}

R ESEARCh by NASA to develop advanced subsonic transport concepts and technologies is aimed at meeting or Rexceeding aggressive targets for reductions in fuel burn, emissions, and noise on aircraft entering service in the Mid Term time frame between 2025 and 2035, as well as the Far Term beyond 2035. These targets have generated sustained interest in prediction methods capable of quickly assessing each technology's impact as it continues to mature. The Mid Term goals outlined in the NASA Strategic Implementation Plan [1] are to reduce cumulative noise by 32-42 dB relative to the FAA's Stage 4 limits, reduce $\mathrm{NO}_{x}$ emissions by $80 \%$ relative to CAEP 6, and reduce fuel consumption by 50-60\% relative to a 2005 best in class aircraft. The drive to develop ultra-efficient vehicles has led to the consideration of configurations outside of the scope of legacy aircraft designs and, by extension, legacy empirical based prediction methods. The results of this departure from configurations whose prediction can be tempered by experimental results are twofold. There is a need to formulate parametric physics-based prediction methods that are not limited by experimental databases, as well as a need to assess the uncertainty in using a particular prediction method for a given configuration. This will allow for more flexibility in design, and a better understanding of the accuracy and limitations of the prediction. The focus herein is on continuing to improve uncertainty quantification of predictions made using the Aircraft NOise Prediction Program (ANOPP). Work on uncertainty quantification began under the Environmentally Responsible Aviation (ERA) Project [2, 3], and continues as part of the Advanced Air Transport Technology (AATT) Project, in an effort to better understand and improve the reliability of predicting the noise of unconventional aircraft configurations. Specifically, the improvements are related to the uncertainty of the individual elements (e.g., fan noise, jet noise) that are propagated to the system level to quantify the uncertainty of the vehicle noise.

The value of understanding the prediction uncertainty is multifaceted. One such application of the system noise uncertainty results is to vision vehicle configurations that AATT has identified as potential configurations for implementing several key technologies to reach the Mid or Far Term goals. At the system level, the uncertainty in the prediction is a key input for determining the risk of a vision vehicle failing to achieve the predicted performance. It is unlikely that industry will consider an aircraft configuration change unless the prediction model matures to the point where the uncertainty in the estimated noise benefit is low enough to accept the risk of changing architectures. If there is large uncertainty in the prediction, then further refinement of the prediction and understanding of the technology are warranted to ensure realization of the desired performance. This also holds true at the element level, where uncertainty in the prediction of the overall system stemming from that element allows for a quantitative means of assessing the risk in depending on a technology to aid in reaching the overall goal. This can help to set priorities where resources for risk reduction testing are limited, and aid in refining roadmaps [4] for reaching the Mid Term goals. This can also help 
identify element prediction models that need to be improved to confidently steer research and resources earlier in the life cycle of the project toward technologies that are most likely to result in a noise benefit.

Thomas et al. [3] introduced a process for quantifying the uncertainty in the system noise of an aircraft by estimating the uncertainty in each of the individual elements, and propagating the elemental uncertainties to the system level in a direct Monte Carlo approach. These elements are of three types: sources, noise reduction technologies, and propulsion airframe aeroacoustic (PAA) effects. Uncertainties were assigned to each element based on the best available information, and the system level uncertainty was estimated using a Monte Carlo uncertainty propagation method. Differences in the fidelity of data available for each element resulted in a range of approaches for determining the element uncertainties. The depth of quantitative analysis for some of these approaches is limited by a lack of experimental data. This analysis followed the progress of the prediction uncertainty over the course of the ERA project, with an initial assessment in 2009, an interim assessment in 2013, and a final assessment in 2016. Over this time, the maturation of the element prediction models and approach for determining the uncertainty resulted in a reduction in the standard uncertainty of the cumulative certification noise from 4 EPNdB in 2009 to 2.4 EPNdB in 2016. These correspond to $95 \%$ expanded uncertainties of $\pm 8 \mathrm{EPNdB}$ and $\pm 4.8 \mathrm{EPNdB}$, respectively.

As mentioned previously, there is a need for generating physics-based system level noise predictions that can quickly estimate the noise of the aircraft. To the knowledge of the author, there are no aircraft prediction codes that predict all of the sources and interaction effects using purely physics-based models. However, there are several codes that employ a mixture of empirical and physics-based prediction methods, including ANOPP and ANOPP2 [5] at NASA, the Parametric Aircraft Noise Analysis Module (PANAM) [6] at the German Aerospace Center (DLR), and the Ray Acoustics Code for Noise Modeling and Estimation (CARMEN) [7] at the French Aerospace Lab (ONERA), to name a few. Each of these institutions has different approaches and applications for their system noise tools. However, interest in understanding the performance of each of the codes in relation to each other as well as model and full scale experiments has culminated in the formation of the Aircraft Noise Simulation Working Group (ANSWr). One of the focuses of this group is to better quantify the uncertainty of these tools. This will allow for more meaningful comparison between predictions of the same aircraft with different codes, as apparent disagreements may be due to the uncertainties in the techniques being used.

Although work continues in each group, the only published work to date, apart from the previous study [3] that this paper aims to update, is a recent paper from DLR [8]. That work focused on estimating the uncertainty of conventional tube-and-wing aircraft using a sensitivity-based uncertainty propagation technique as outlined in the Guide to the expression of Uncertainty in Measurement (GUM) [9]. The context of the DLR paper was preparation for comparison with existing full scale flight data. For that application, additional uncertainty sources were considered that are outside the scope of this paper. These include uncertainties in the true flight path, atmospheric conditions, engine state, or control surface deflections. These additional sources of uncertainty from the inputs and propagation through the atmosphere were noted to exceed the model uncertainty in some cases. While these are important to include in the context of prediction code validation with flight test, they are not applicable to assessing the noise performance of a given aircraft concept. For consistency in comparing the noise performance of several aircraft concepts, it is preferable to focus solely on the uncertainties stemming from modeling, while maintaining consistent atmospheric conditions and assuming that the aircraft follows the simulated flight path in a deterministic fashion. The uncertainty in the DLR prediction was also carried out over an area of ground to form a contour map of the uncertainty, rather than simply the certification points, which was the objective of Thomas et al. [3]. Again, this is due to differences in motivation, with the DLR paper using this result in the operational application of ground observers being awakened, while the NASA papers focus on the uncertainty of predicting conceptual designs of advanced, future aircraft.

As the uncertainty quantification process is application dependent, this paper aims to provide sufficient context and details of the procedure used for the hybrid wing body concept. The purposes of the work are to:

- describe the refinements in the element uncertainty calculation procedure,

- illustrate which sources of uncertainty are included through a detailed example of the jet noise,

- account for updates in the airframe noise prediction methods, and

- compare with previous results to identify the impact of the updated uncertainty procedure and prediction methods. Based on the results, direction is then provided for the focus of continued improvements.

\section{Aircraft Configuration}

The uncertainty quantification described in this paper is focused on a hybrid wing body (HWB) in the large twin aisle aircraft class, sized for 301 passengers [10]. An artist's rendering of the aircraft is shown in Fig. 1. The general 


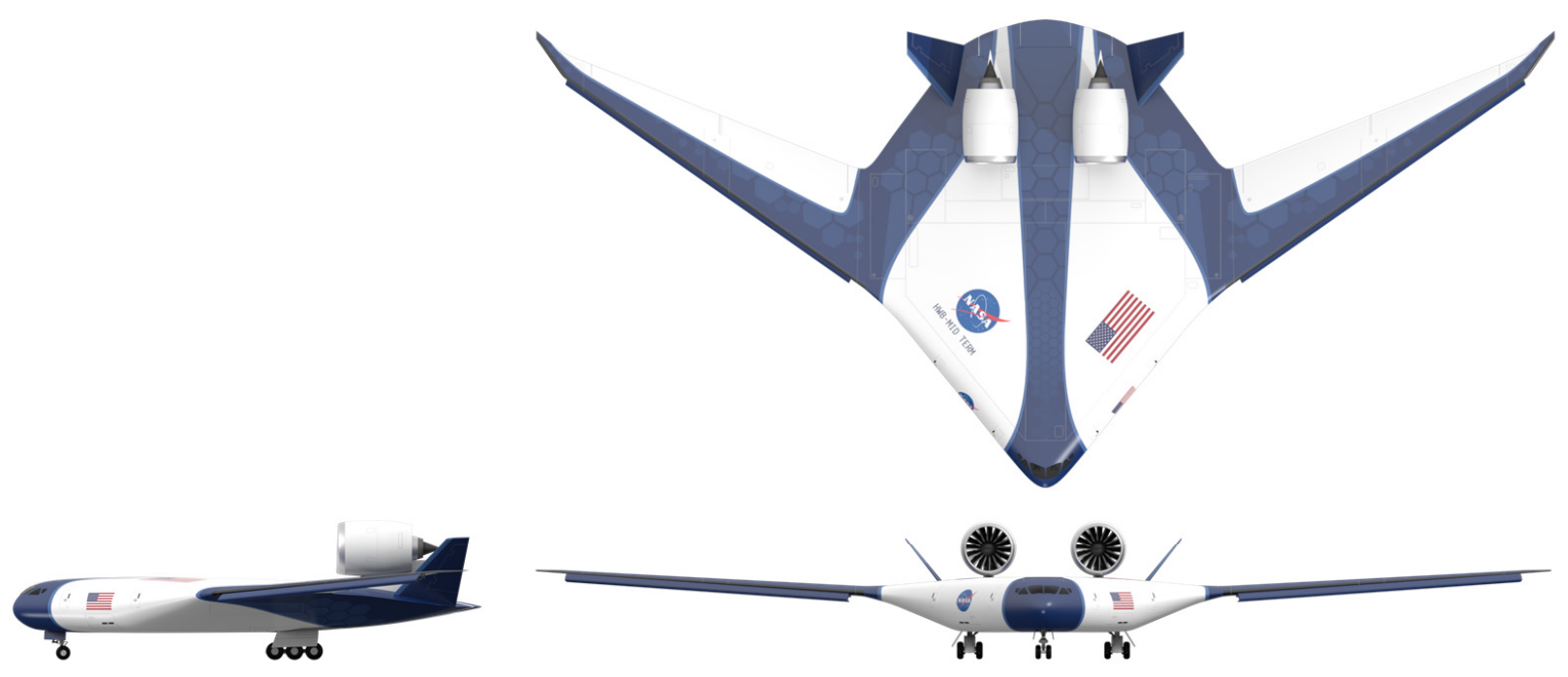

Fig. 1 A standard three view of the HWB301 is shown.

aspects of this design are the same as has been used in previous works [3, 4], and some relevant parameters are presented in Table 1. As mentioned, the actual aircraft definition is identical to the HWB-2016 from the end of ERA. The aircraft is equipped with technologies which are expected to be mature enough for commercial applications in the Mid Term time frame. The airframe integrates technologies like Krueger flaps to enable laminar flow wings and damage arresting composites. The engines are ultra high bypass geared turbofans (GTF) with short inlets, which allow for lower fan speeds and higher efficiency while minimizing the cost of the additional weight and drag of the larger engine. Several improvements to the prediction process have been made since previous published predictions. These improvements are discussed in Section III and are also described in previous work [4], where the configuration used in the prediction here is the same as configuration C6 in the previous work. Within this paper, reference to the HWB301 will be in regards to this configuration.

Table 1 Summary of Relevant Aircraft Parameters.

\begin{tabular}{lrc}
\hline Dimension & Value & Units \\
\hline Passengers & 301 & \\
Range & 7500 & $\mathrm{NM}$ \\
Operating Empty Weight & 253806 & $\mathrm{lb}$ \\
Payload & 118100 & $\mathrm{lb}$ \\
Total Fuel & 163258 & $\mathrm{lb}$ \\
Takeoff Gross Weight & 535164 & $\mathrm{lb}$ \\
\hline
\end{tabular}

\section{Noise Prediction Details}

In order to predict the radiated noise of the HWB301, the ANOPP code is used, running within a framework provided by ANOPP2. This process, outlined in Fig. 2, allows for the incorporation of several data and modeling sources of varying fidelity. The research version of ANOPP is used for all of the noise predictions in this paper. The research version contains several data sources and prediction models that are not yet part of the publicly released version of ANOPP. The data sources are identified in the figure highlighting the prediction process as experimental data inputs, stemming from work carried out in the past several years [11-15]. These data are incorporated into the prediction in several ways. The first is within the process used to generate the conceptual design of the aircraft. Based on the mission and overarching constraints on the general aircraft design, an aerodynamic system analysis and optimization of the design is carried out. This results in a defined aircraft concept and a realistic flight path. Information about the engine 
state is generated using the Numerical Propulsion System Simulation (NPSS) [16]; the flight path is generated using the FLight OPtimization System (FLOPS) [17]; and estimates of the performance are obtained using a modified vortex lattice (MVL) approach [18]. The geometric definition of the aircraft, engine state information, and flight path are then used as inputs to the noise prediction process.

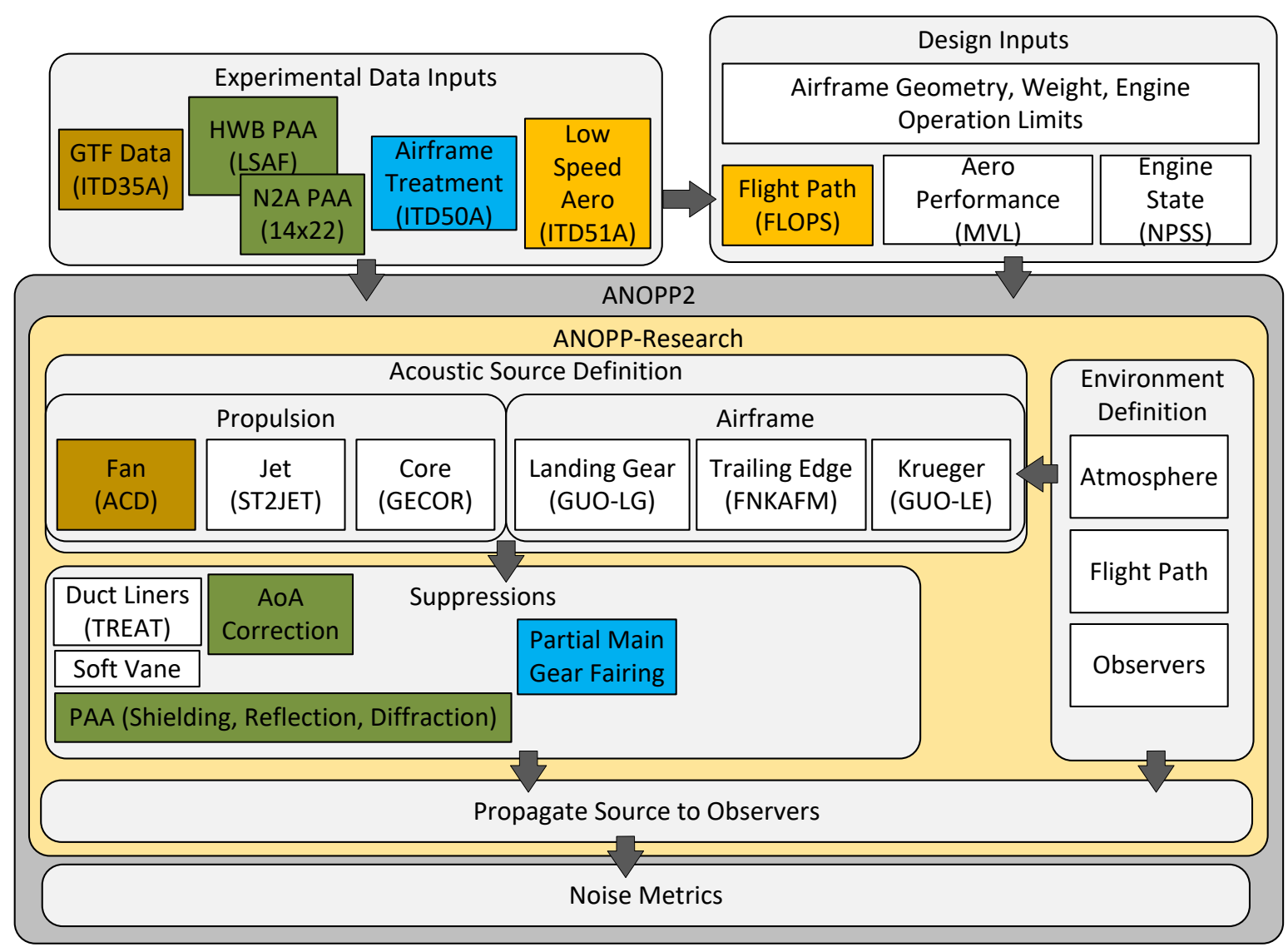

Fig. 2 The overall data flow for the noise prediction is shown. The heritage of the data is shown through consistent coloring. Suppressions are applied to the source modules above them. Names of the software or ANOPP modules used to generate the prediction are given in parentheses.

Within ANOPP, each of the acoustic sources is defined using a combination of the previously specified information and the experimental data inputs. With regard to the prediction of propulsive noise, measured GTF data are scaled and used to predict the fan noise source. The jet noise is modeled using an updated version of the Stone jet model [19], and the core noise is modeled using GECOR [20]. With respect to the airframe noise, the gear are modeled using the Guo landing gear method [21]; the Krueger noise is modeled with the Guo leading edge method [22]; and trailing edge noise is modeled using the Fink airframe model [23]. Shielding effects are accounted for using suppressions based on previous wind tunnel experiments in the Boeing Low Speed Aeroacoustic Facility (LSAF) [12] and the NASA Langley Research Center 14- by 22-Foot Subsonic Tunnel [13].

Several modeling changes were made since the previous study regarding uncertainty [3], and more detailed descriptions of the changes can be found in Thomas et al. [4]. These include:

- corrections to the values used for the gear length exposed to the flow,

- refined values of the elevon deflections,

- changes in the resulting shielding mapping,

- improved estimate of the interstage liner effectiveness,

- changes to the treated bifurcation area, 


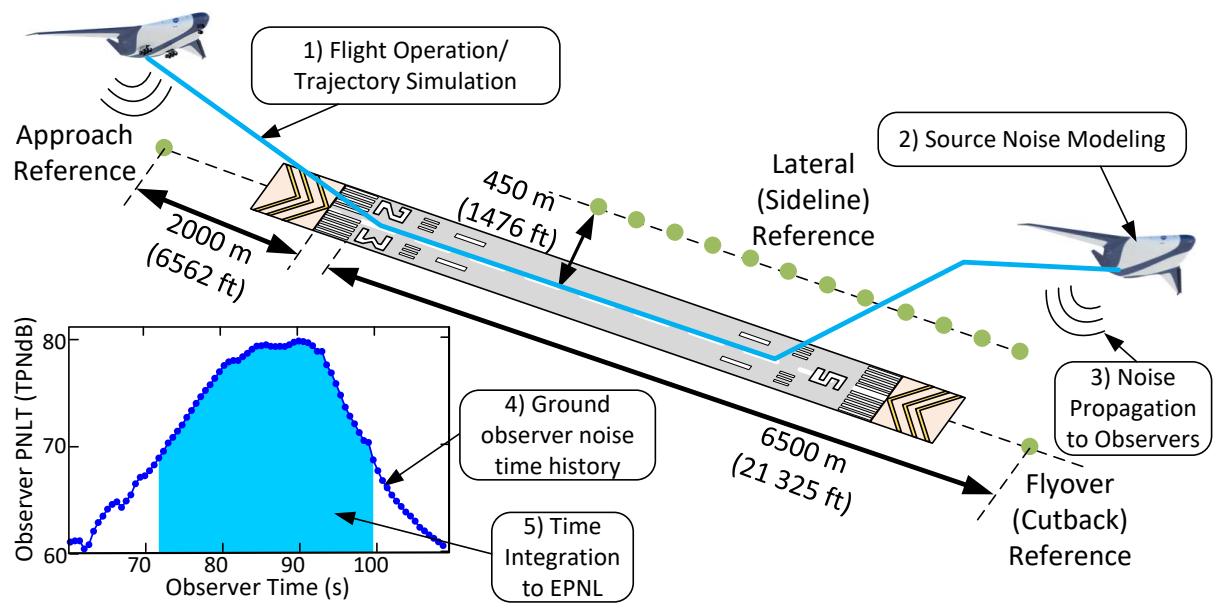

Fig. 3 The location of the certification points along with the high level process for obtaining an EPNL from a prediction.

- tuning of the inlet and aft liner treatment to the fan source, and

- modeling updates for the Krueger flap bracket noise reflection.

Three additional updates have been made since publication of Thomas et al. [4]: a change to the liner module to reflect a more realistic attenuation from multi-degree of freedom liners, a correction to the atmospheric propagation method to follow that of SAE ARP 866A [24], and a change in the options used in the Stone jet model [19] that improved agreement with an extensive jet noise wind tunnel database.

After the noise sources are defined and modified to account for the PAA effects and noise reduction technologies, the source spectra are specified at one-third octave band resolution that can change as a function of time in the prediction, and propagated to the observer. In general, the observers can be placed at any spatial location, but the primary focus of the work here is in relation to the received noise at the certification points. These points, defined by the Code of Federal Regulations Title 14, Part 36, are shown in Fig. 3. Utilities within ANOPP2 are used to compute the effective perceived noise level (EPNL) at each of the observer locations.

\section{Uncertainty Quantification Details}

An overview of the general process that is used in determining the uncertainty at the system level is shown in Fig. 4. The first column in the process highlights the steps taken in setting up the prediction and Monte Carlo method. The noise prediction elements are identified based on the aircraft configuration, and the most appropriate method of predicting that element is chosen. Following that, the method for establishing the uncertainty is chosen based on the particular element. Three of the four methods used in Thomas et al. [3] are used here, namely the formulation, reference data prediction test, and inferred methods. These are chosen based on the type of data that are available to validate each of the element prediction methods. The formulation method is typically applied to the physics-based prediction methods, while the reference data prediction test method is generally chosen for semi-empirical methods. The inferred method is chosen when there is not sufficient quantitative comparison to allow for the previous two methods. For the reference data prediction test method, there is a hierarchy for determining which data are preferred for use in the comparison. From highest to lowest fidelity, the classes are:

- Full scale flight data,

- Subscale, system level experimental data,

- Subscale, subsystem level experimental data, and

- Subscale, isolated element experimental data.

These classes are separated by the level at which they integrate effects which might not be present in isolation, providing a better overall notion of how a prediction will compare with a full scale vehicle where these integrated effects are 
important.

Based on the uncertainty method and associated supporting data, uncertainties are quantified at the element level. This leads to the second column, which illustrates the Monte Carlo method itself. The element level uncertainties are propagated by directly predicting the noise at the system level for blocks of 250 realizations randomly sampled from the associated element probability distributions. The collection of predictions is then used to generate a statistical distribution of the output variable, the EPNL at each of the certification points, as well as a cumulative level. This process is repeated until all of the statistics are converged to within a desired tolerance for the entire block. The convergence tolerance for the mean and standard deviation levels is set such that the results can be displayed to two decimal places.

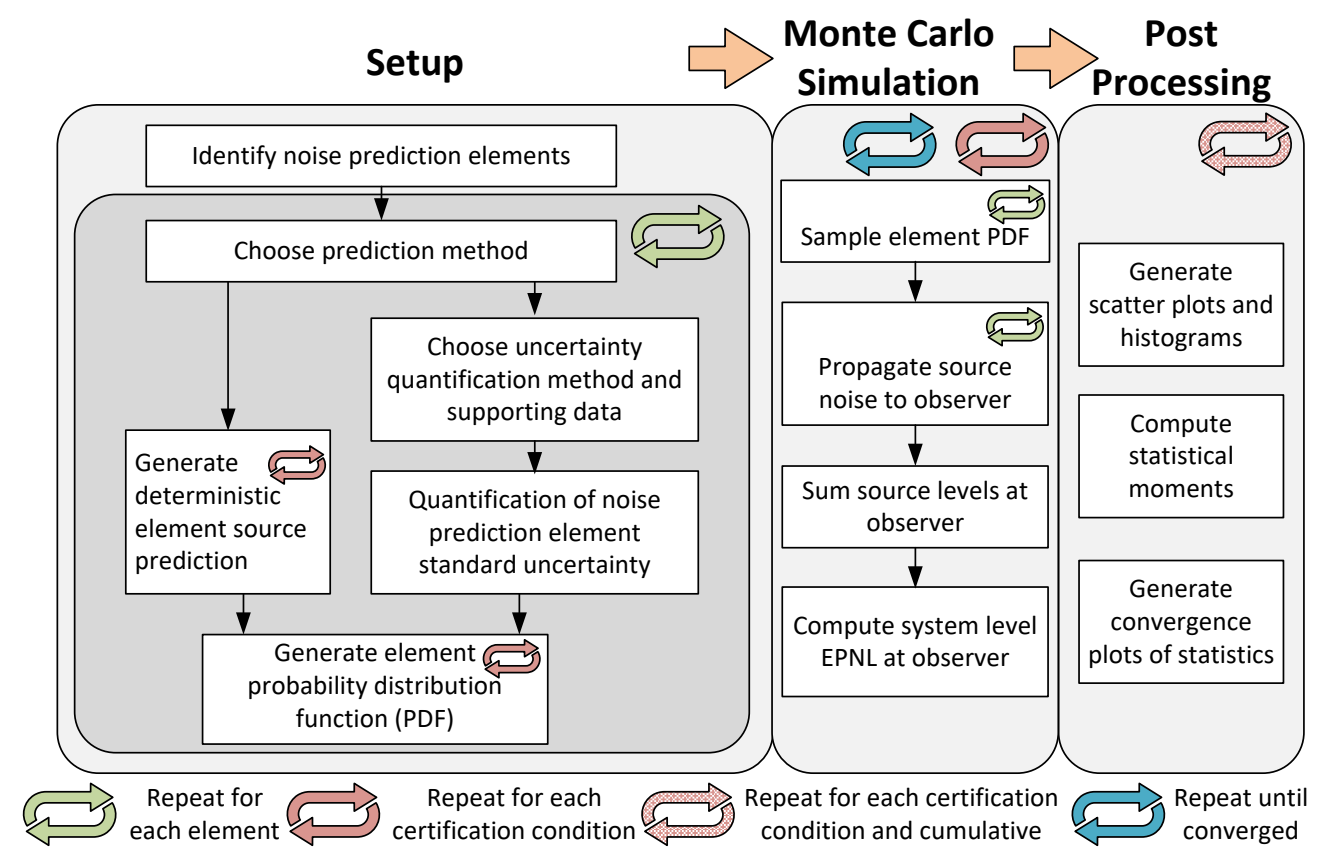

Fig. 4 The framework for the Monte Carlo method as applied to the system noise prediction is shown.

As with any technical discipline, it is important to ensure the proper meaning is conveyed by consistent terminology and processes. Whenever possible, this paper strives to follow the definitions in the International Vocabulary of Metrology (VIM) [25], and procedures defined in the GUM supplement on the Monte Carlo method [26]. Unless otherwise noted, uncertainties are presented as standard uncertainties, and coverage intervals (CIs) are probabilistically symmetric with a $95 \%$ coverage probability.

\section{A. Methodology and Nomenclature}

In order to quantify the uncertainty in each of the element predictions, the sources of uncertainty within the element must first be identified. The three uncertainty quantification methods used in this paper have differences in how these uncertainties are estimated, but all three aim to be consistent with regards to which uncertainties are included. Details of the formulation method can be found in Guo et al. [21]. The inferred method is applied based on experience, any available data, and input of subject matter experts in the field. The process for the reference data prediction test method is contained within this section of the paper.

For a simulated result, uncertainties come from three general sources: inputs, numerics, and modeling assumptions [27]. The input uncertainties come from any variable that the model uses to return a result. Numerical uncertainty arises from several sources including truncation and rounding errors that accumulate over the course of a simulation. Finally, assumptions in the model itself produce uncertainty, resulting from incomplete physics or inaccuracy in empirical modeling constants. For prediction of aircraft concepts in the conceptual design stage, such as the HWB301 considered here, the aircraft design and flight path are considered fixed, and no input uncertainty exists. Therefore, the 
uncertainty of the element prediction at full scale can be estimated as

$$
u_{P, F S}=\sqrt{u_{M, F S}^{2}+u_{N, F S}^{2}}
$$

or the root sum square of the full scale modeling and numerical uncertainties. For predictions of the type performed in ANOPP, the numerical error is dominated by the resolution to which the results are displayed to the user, typically one to two decimal places, rather than the precision to which the calculations are performed. This numerical uncertainty is expected to be much less than the contributions from the modeling uncertainty, but is included for completeness. Therefore, only estimation of the modeling uncertainty is needed to determine the uncertainty in the element prediction. However, estimation of the modeling uncertainty requires some information with the full physics of the process, and full fidelity of the design, which is unavailable for conceptual aircraft. Even for existing aircraft, there are challenges in obtaining this information, either from flight test data, or a simulation which completely resolves all of the relevant scales of the problem. Instead, the modeling uncertainty of the full scale element, $u_{M, F S}$, is estimated from an estimate of the modeling uncertainty of a subscale element, $u_{M, S S}$. In general, there are multiple scales which contribute to noise generation on an element, and scaling from subscale to full scale introduces an additional source of uncertainty, $u_{S}$. The modeling uncertainty of the full scale element is

$$
u_{M, F S}=\sqrt{u_{M, S S}^{2}+u_{S}^{2}}
$$

where the assumption of uncorrelated uncertainties results in the addition in the root-sum-square sense. It then remains to estimate the uncertainty in the scaling, as well as the modeling uncertainty at subscale.

The validation approach outlined by Coleman and Steele [27] is utilized to quantify the subscale modeling uncertainty. This process allows for quantification of the modeling uncertainty, through comparison between the modeled physics of a simulation, and an experimental result that captures the full physics. This is illustrated in Fig. 5. The same uncertainty sources (e.g., input, numerical, and modeling), must be considered for the subscale comparison independent of their inclusion at full scale. Again at subscale, ANOPP simulations have numerical errors which are dominated by the number of digits output to the user. However, the contributions of input uncertainty cannot be neglected at subscale. This difference stems from predicting an abstract aircraft element for which the design is considered certain at full scale, while predicting a concrete aircraft element which has some uncertainty in the true geometry and flow conditions of the subscale experiment which are then used to perform the prediction. The subscale modeling error is estimated from a comparison between the prediction and experiment as outlined below.

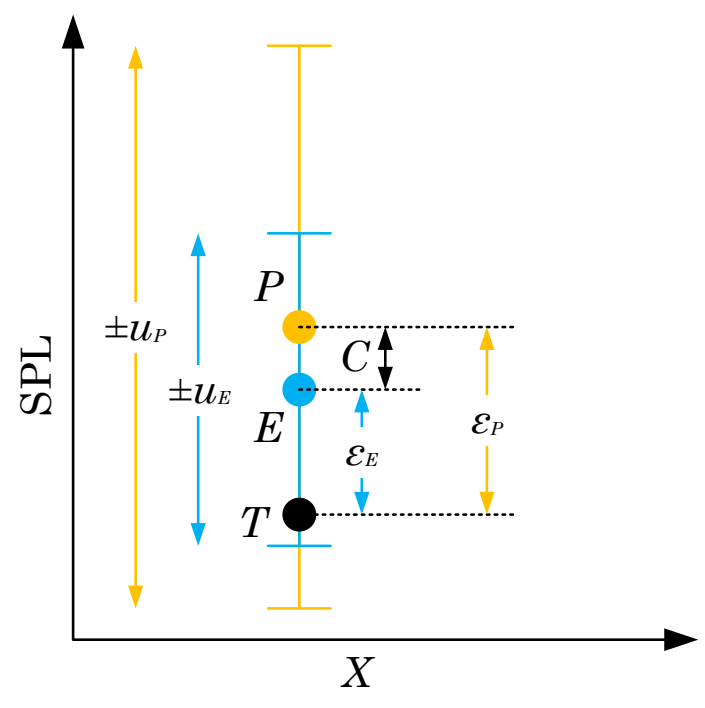

Fig. 5 The validation by data approach is shown. Errors are denoted by $\varepsilon$, and standard uncertainties by $u$. Figure adapted from Coleman and Steele [27].

The validation approach applied to a single point begins by determining the comparison error, or difference between the prediction and experiment. If, for a moment, the true value is assumed to be known, the signed comparison error 
between the experiment and prediction at a single point is related to the signed error in the prediction and experiment as

$$
C=P-E=\varepsilon_{P}-\varepsilon_{E},
$$

where the prediction error is a sum of the input, numerical, and modeling errors,

$$
\varepsilon_{P}=\varepsilon_{I}+\varepsilon_{N}+\varepsilon_{M}
$$

Combining Equations 3 and 4 results in an equation relating the modeling error to all of the other constituent errors. Since the true value is not known, the modeling error cannot be directly obtained. Instead of the modeling error, the modeling uncertainty can be estimated as

$$
u_{M, S S}=C \pm \sqrt{u_{E, S S}^{2}+u_{I, S S}^{2}+u_{N, S S}^{2}}=C \pm u_{m}
$$

the sum of the comparison error with the combined measurement uncertainty, $u_{m}$. The definition of $u_{m}$ is one of convenience for the following equations, rather than one out of the particular importance of that quantity. Equation 5 makes it clear that the modeling uncertainty can only be validated to the uncertainty of the experiment, placing importance on comparison with high quality experiments. Further, this uncertainty estimate is only strictly valid at the operating conditions under which the validation is performed, highlighting the need for experiments that are tailored as closely as possible to the particular configuration of the simulated aircraft. This minimizes the amount of uncertainty which enters the process when the subscale results are extrapolated to full scale. This process improves upon the previous work [3] in that the experimental uncertainty and input uncertainty are now included, whereas previously only the comparison error was considered.

Retention of uncertainty information for an element as a function of the relevant independent variables (i.e., frequency, emission angle) can be more cumbersome to integrate into a Monte Carlo method, and, as it is based off a single data point (operating condition), is sensitive to any outliers that may be present. To counter this, a weighted averaging process is carried out across the independent variables to determine a single representative uncertainty that can be applied to ease implementation and minimize the impact of outliers. This can also be extended to average over multiple operating conditions where experimental data are available. This averaged subscale modeling uncertainty is then

$$
\hat{u}_{M, S S}=\hat{C} \pm \sqrt{\hat{u}_{m}^{2}+\hat{u}_{C}^{2}+\hat{u}_{u_{m}}^{2}},
$$

where $\hat{C}$ and $\hat{u}_{m}$ are the weighted average comparison error and measurement uncertainties, while $\hat{u}_{C}$ and $\hat{u}_{u_{m}}$ are the corresponding uncertainties of those mean quantities. The standard uncertainties $\hat{u}_{C}$ and $\hat{u}_{u_{m}}$ are estimated as the standard deviation, rather than the standard deviation of the mean (the sample standard deviation divided by the square root of the sample size). This is a conservative estimate of the mean subscale modeling uncertainty, reflecting the potential for correlation between samples of $C$ or $u_{m}$ at different conditions. Once the average subscale modeling uncertainty is computed, this is extrapolated to full scale using Equation 2. An example of this process, which replaces a more qualitative past approach is described in Section IV.B.

After the uncertainty of an element has been estimated from comparison with experiment, a sample probability distribution must be assigned. Several probability density functions (PDFs) are assumed, depending on the type of element. In the previous assessment all noise source elements and the MDOF liner uncertainty were assumed to follow a Gaussian PDF. Noise reduction technologies and PAA effects were prescribed a one-sided Gaussian PDF, defined by

$$
p(x)= \begin{cases}\frac{2}{\sqrt{2 \pi} \sigma} \exp \left(-\frac{(x-\bar{x})^{2}}{2 \sigma^{2}}\right) & x \geq \bar{x} \\ 0 & x<\bar{x} .\end{cases}
$$

This distribution was chosen as it is representative of the likelihood that the noise reduction technology will be less effective when implemented on a full scale flight vehicle than the subscale experiment from which it was derived. More directly, the underlying noise reduction technology was assumed to have a Gaussian distribution, and the half of the distribution corresponding to noise reduction in excess to that seen in subscale testing was removed to account for scaling effects. For the PAA maps, this distribution was chosen for the additional reason that the maps were obtained from experiments with a broadband source, which may have more noise reduction from shielding than a tonal source. The noise reduction technology realizations were also tailored to ensure that they did not produce a net noise increase due to the presence of the technology. Each realization of the noise reduction technology is the sum of the deterministic 
prediction and a perturbation. When the perturbation exceeds the deterministic prediction, there will be a net noise increase. To avoid this, a limit was placed on the positive tail of the one-sided Gaussian distribution. A similar constraint was placed on the PAA maps from the physical limit that the presence of a shielding surface will not produce a net increase in noise. There was also a restriction placed on the samples for all elements to be within two standard deviations of the mean to prevent the lowest probability occurrences.

The current assessment is similar but does have some significant differences. All noise source elements and the MDOF liner uncertainty are still assumed to follow a Gaussian PDF. The first difference is that the restriction that the samples be within two standard deviations was removed, as there is not a strong physical reason for retaining it. The second is that for the noise reduction technologies and PAA maps, the one sided Gaussian has been replaced by an exponential distribution

$$
p(x)= \begin{cases}\frac{1}{\sigma} \exp \left(-\frac{x-\bar{x}}{\sigma}\right) & x \geq \bar{x} \\ 0 & x<\bar{x},\end{cases}
$$

which has been used in the past when information about the distribution is limited to knowledge of a best estimate of the deviation $\sigma$ and the constraint that $x$ must be greater than some lower limit. Fig. 6 shows the PDF and cumulative distribution function (CDF) of both types of distributions. The PDFs are normalized by $\sigma$ in both cases so that the entire family of distributions can be shown in a single plot. It is important to note that while there is a higher probability density near the best estimate $\bar{x}$ for the exponential distribution, it also has a tail that is significantly more positively skewed than the one-sided Gaussian distribution. This is reflective of the increased confidence in the best estimate, paired with the likelihood that integration of an effect or technology at the system level will exhibit reduced effectiveness.
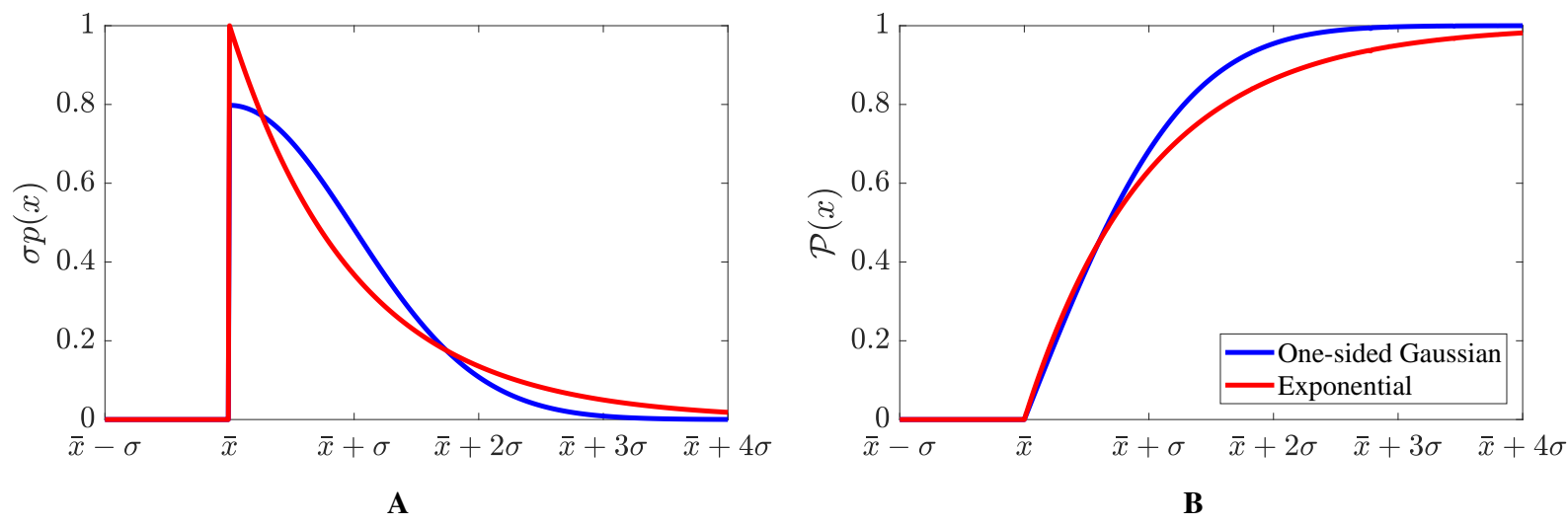

Fig. 6 Normalized candidate PDFs are shown for the one-sided Gaussian and exponential distributions, A, as well as CDFs for each distribution, $B$.

\section{B. Jet Source Noise Uncertainty: An Example}

The process used to determine the uncertainty of the jet noise element is detailed as an example of the reference data prediction test method, which has been refined since the last publication [3]. This process corresponds to the two middle boxes in the right column of the Setup section of Fig. 4, which is repeated for each element using its respective uncertainty estimation method. The first step of the reference data prediction test method is to identify an experiment that is as close as possible to the configuration to be predicted. In the previous assessment, the element prediction uncertainty was then determined from a visual estimation of the comparison error. By contrast, the prediction and experiment are now compared quantitatively in order to determine the element prediction standard uncertainty. Through this comparison, an average subscale modeling standard uncertainty is determined from Equation 6, and can be used in conjunction with an estimate of the scaling uncertainty and Equations 1 and 2 to obtain a full scale prediction standard uncertainty, $u_{P, F S}$. A Gaussian distribution for the jet noise element is built with the mean coming from the deterministic jet prediction and the standard deviation equal to $u_{P, F S}$. The distribution is sampled during the remaining steps of the Monte Carlo simulation to determine the standard uncertainty of the vehicle at the system level. It should be made clear that the uncertainty in the prediction will vary based on the specific aircraft configuration and technology implemented. Specifically, for the jet noise uncertainty estimate, the numbers presented herein are not directly applicable 


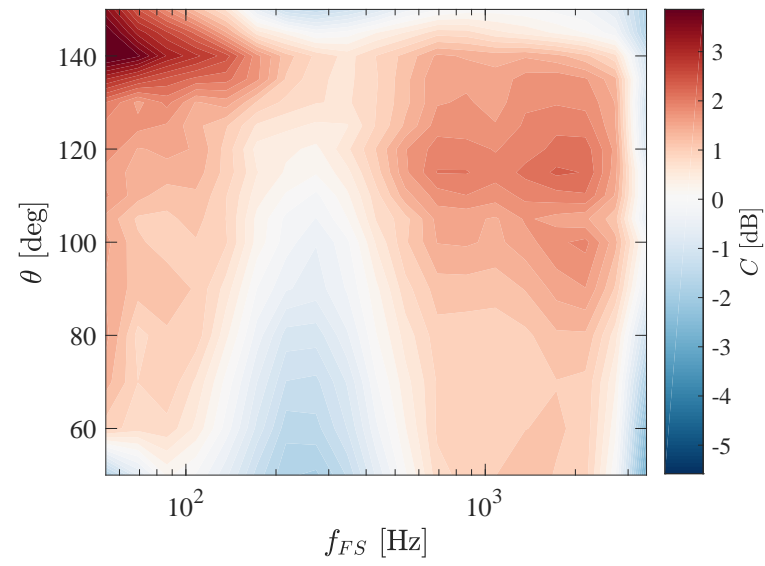

A

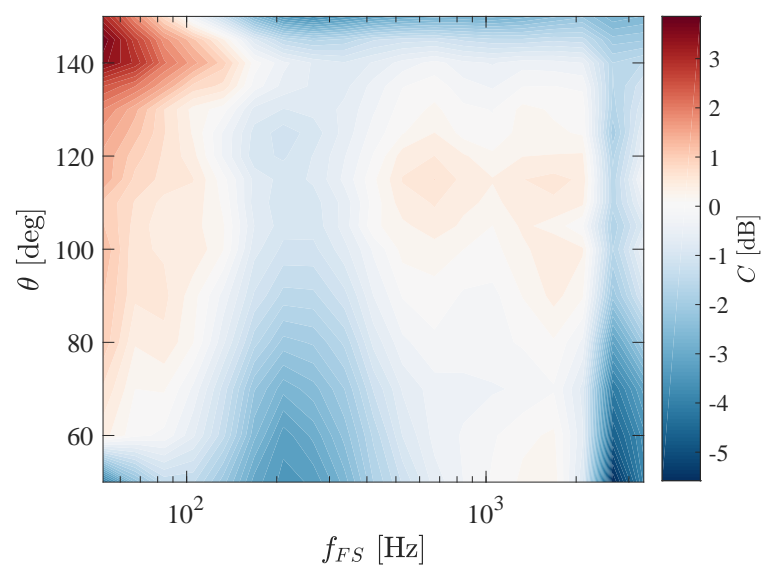

B

Fig. 7 The comparison error for the sideline, A, and cutback, B, experimental conditions and the Stone jet prediction in dB SPL are shown for a freestream Mach number of 0.2 as a function of polar angle and full scale frequency.

to other unconventional configurations unless they, too, have a similar engine, operating under the same conditions.

The experimental data that are chosen as representative of the jet noise on the HWB301 were taken in the Boeing Low Speed Aeroacoustic Facility (LSAF), on a $5 \%$ scale nozzle, relative to the HWB301, with a nominal bypass ratio (BPR) of 15. The jet is axisymmetric, and the core flow is heated to match realistic design conditions for the temperature and pressure ratios for the full scale HWB. The LSAF is an anechoic open jet facility that has taken special care to identify and reduce extraneous rig noise [28], allowing for the capability to obtain detailed aeroacoustic measurements of hot jet experiments carried out under simulated flight conditions. The experimental standard uncertainty for the acoustic measurements in the facility is estimated by combining the uncertainties stemming from repeatability in the test condition, microphone distances, atmospheric conditions, and microphone calibration errors. For the measurement campaign that is chosen, experimental Mach numbers of 0.17 and 0.2 are available, with 0.2 being equivalent to the approach Mach number for the HWB301, and slightly lower than the Mach number for the lateral and flyover positions of 0.25. Two engine conditions are available at each Mach number, with one representative of the engine cycle at cutback and the other at sideline.

With the experimental cases chosen, the next step is to run a prediction of the exact experimental setup at the jet element level using the same model used in the full aircraft prediction, with all of the same options and user settings. This is done using the updated version of the Stone jet model, ST2JET. A comparison of the prediction and the experimental data is shown in Fig. 7 for a representative cutback and sideline condition. There is good agreement between the model and the experiment, typically within a $2 \mathrm{~dB}$ range of each other, with some disagreement near the edges of the domain. In general, this comparison should be carried out between the experiment after it has been scaled to full scale, along with the full scale prediction, to be as relevant as possible to the HWB301 prediction. However, experimental data would not scale to standard one-third octave bands unless the scale factor was an integer power of two, which would require energy to be split between adjacent bands for comparison with the full scale prediction which yields results at the standard one-third octave bands. Fortunately, the Stone jet model scales with jet area alone, and as a relative measure, the comparison error values do not change with scale; only the corresponding frequency to which these error values are mapped changes with scale. The comparison is therefore carried out at subscale to avoid any issues associated with splitting energy between neighboring one-third octave bands, and then scaled to full scale as one-third octave bands with nonstandard center frequencies. These predictions allow for determination of the average comparison error, which is discussed more thoroughly below. Separately, the uncertainty due to input uncertainty and numerical error must also be tabulated. The numerical error is straightforward; the results of the simulation are reported to the user with two decimal places, so the expanded uncertainty is $\pm 0.005 \mathrm{~dB}$. The uncertainty of the inputs is computed by first estimating uncertainty in each of the geometric and flow parameters that is used in the jet noise model and propagating that uncertainty using a separate Monte Carlo simulation of the jet element alone and running until convergence. The propagated uncertainty is then used as the uncertainty of the inputs, $u_{I, S S}$. This input standard uncertainty can be 
combined with the experimental standard uncertainty and numerical standard uncertainty to determine the combined measurement standard uncertainty.

As the comparison error and combined measurement uncertainty are available over a range of frequencies and angles, this results in a functional dependence of the comparison error, and in turn the prediction uncertainty, in both frequency and angle. However, retention of this information and implementation in the Monte Carlo simulation is much more cumbersome than simply using a zeroth order approach where the uncertainty is estimated by a single representative number rather than varying as a function of angle and frequency. This approach still incorporates all the data over the full frequency and angular ranges by determining the single number comparison error with a weighted averaging process that is indicative of the error most closely associated with the frequencies and angles of interest. This allows for straightforward implementation of the resulting estimate of the modeling uncertainties in the Monte Carlo procedure, and minimizes the effect of any potential experimental outliers. So long as care is taken to choose this number appropriately, this simplification should yield results that are sufficient for configurations in the conceptual design stage.

The output uncertainty of the Monte Carlo simulation of interest is the vehicle EPNL, with units EPNdB, which accounts for several effects in the frequency content and amplitude of a signal to give a single number representative of the noise of the source. In a similar fashion, the elemental standard uncertainty estimates used as inputs to the Monte Carlo simulation should also be reflective of the EPNL at the observer if only a single number is used. This implies that the single number elemental standard uncertainty should more closely consider the subset of frequencies and angles that will have an impact on the observer EPNL. Two weighting factors are used to account for the amplitude of the received signal at the observer and a third accounts for the spectral character. The first weighting factor for the uncertainty, $w_{p^{2}}$, is the source level on a pressure squared basis of the prediction at a given frequency and angle, so that uncertainties at frequencies and angles with more energy are weighted more heavily. The second weighting factor for the uncertainty, $w_{\theta}$, takes into account the variation in distance between the aircraft and observer. For the jet, this weighting is $\sin ^{2}(\theta)$, which accounts for the change in the received level of the source at the observer due to spherical spreading in level overhead flight. This approximation does not take into account atmospheric attenuation, the aircraft attitude, or for the specific case of the lateral certification point, the distance between source and observer due to the offset flight path. However, the correction is, in general, representative of the intended effect, and can be implemented without requiring knowledge of the actual flight path. In order to take the spectral character into account, a third weighting factor, $w_{D}$, is formulated from D-weighting the signal in lieu of the procedure for computing EPNL. It should be noted that the D-weighting is based on the full-scale frequencies, not subscale. The overall normalized weighting is

$$
w_{n}\left(f_{i}, \theta_{j}\right)=\frac{w_{p^{2}}\left(f_{i}, \theta_{j}\right) w_{\theta}\left(f_{i}, \theta_{j}\right) w_{D}\left(f_{i}, \theta_{j}\right)}{\sum_{i} \sum_{j} w_{p^{2}}\left(f_{i}, \theta_{j}\right) w_{\theta}\left(f_{i}, \theta_{j}\right) w_{D}\left(f_{i}, \theta_{j}\right)} .
$$

The product is taken rather than the sum of the weights, reflecting the way that their combined influence would affect the pressure squared value. The representative weighted mean statistic is then determined from the desired variable at each frequency and angle via

$$
\hat{x}=\frac{\sum_{i} \sum_{j} w_{n}\left(f_{i}, \theta_{j}\right) x\left(f_{i}, \theta_{j}\right)}{\sum_{i} \sum_{j} w_{n}\left(f_{i}, \theta_{j}\right)} .
$$

Similarly, the weighted standard uncertainty of the desired variable is defined as

$$
\hat{u}_{x}=\sqrt{\frac{N}{N-1} \frac{\sum_{i} \sum_{j} w_{n}\left(f_{i}, \theta_{j}\right)\left(x\left(f_{i}, \theta_{j}\right)-\hat{x}\right)^{2}}{\sum_{i} \sum_{j} w_{n}\left(f_{i}, \theta_{j}\right)} .}
$$

This is also incorporated into the elemental modeling uncertainty to account for the uncertainty in estimating the mean uncertainty between the model and experiment. The weighted statistics are computed for the comparison error and combined measurement uncertainty. The relative strength of the weighting factors at each frequency and angle can be compared through a weighting which has been normalized by the average weight,

$$
w_{r}\left(f_{i}, \theta_{j}\right)=10 \log _{10}\left(\frac{w_{n}\left(f_{i}, \theta_{j}\right)}{\overline{w_{n}}}\right) .
$$


A visualization of this relative weight in Figure 8 shows that the weighted statistics will be biased toward the samples at low frequencies and polar angles near $130^{\circ}$ due to the source level, polar angles near $90^{\circ}$ due to minimal spherical spreading losses, and in the $1-4 \mathrm{kHz}$ range due to the D-weighting.

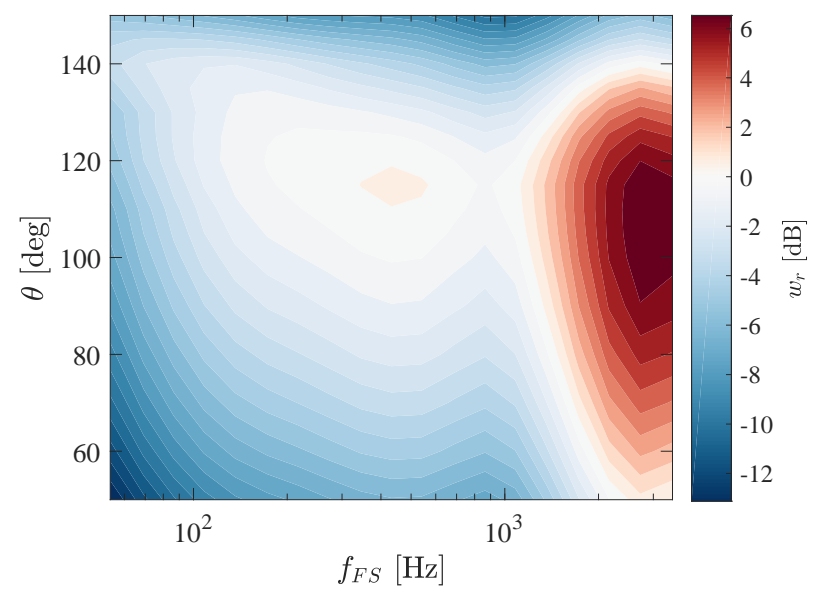

Fig. 8 The weighting for each polar angle and frequency is shown, relative to the average weight over all frequencies and polar angles.

Following the computation of the averaged comparison error and combined measurement uncertainty, the uncertainty due to the scaling process must next be incorporated. For the jet, the assumption of perfect scaling is applied, based on the results of Viswanathan [29] which show that subscale data are representative of full scale jet noise. This means that the full scale and subscale modeling uncertainty are identical, and the full scale prediction uncertainty can be determined using Equation 1.

For the selected experimental cases, the values of the unweighted and weighted estimates of the mean comparison error and combined measurement uncertainty are given in Table 2. Recall that for the comparison error, a positive value represents an overprediction of the data by the model, while a negative value represents an underprediction. Overall, there is not a significant effect on the statistics due to the weighting process. For this reason, it is tempting to state that the weighting process is unnecessary. However, the clear variation of the comparison error with frequency and polar angle in Figure 7 should serve to caution that this situation may not be general to other prediction models or for jet noise predictions of other experiments. This is especially the case for element predictions where the total noise is a composite of multiple source mechanisms. The weighted statistics will focus more on the quality of the comparison for the source mechanism contributing to the noise over a limited range of frequencies, while the unweighted statistics describe the overall agreement. If the quality of some source models is good while others are poor, the unweighted and weighted statistics could differ significantly. These numbers reported here for the comparison error and uncertainty are within the range of values which were reported by Stone [19] for a jet of similar bypass ratio and free stream Mach number, Configuration 7BB. The Stone results are given as a mean comparison error and an rms error which partially accounts for the larger values reported there compared to this paper. There is also a single test point presented by Stone for the same configuration at a Mach number of 0.28 , showing comparison and rms errors which are similar to those presented for the cases at a Mach number of 0.2.

Table 2 Comparison of Jet Standard Uncertainties.

\begin{tabular}{ccrrrrrrrrrr}
\hline Condition & $M a$ & $\bar{C}(\mathrm{~dB})$ & $\hat{C}(\mathrm{~dB})$ & $\bar{u}_{C}$ & $\hat{u}_{C}$ & $\bar{u}_{m}$ & $\hat{u}_{m}$ & $\bar{u}_{u_{m}}$ & $\hat{u}_{u_{m}}$ & $\bar{u}_{P, F S}$ & \multicolumn{1}{c}{$\hat{u}_{P, F S}$} \\
\hline \multirow{2}{*}{ Sideline } & 0.17 & 1.12 & 1.18 & 0.87 & 0.94 & 0.34 & 0.33 & 0.06 & 0.08 & $1.12 \pm 0.91$ & $1.18 \pm 0.98$ \\
& 0.20 & 0.91 & 0.98 & 1.08 & 1.07 & 0.38 & 0.37 & 0.08 & 0.10 & $0.91 \pm 1.12$ & $0.98 \pm 1.11$ \\
Cutback & 0.17 & 0.32 & 0.07 & 1.01 & 1.02 & 0.49 & 0.45 & 0.15 & 0.24 & $0.32 \pm 1.07$ & $0.07 \pm 1.11$ \\
& 0.20 & -0.40 & -0.53 & 1.33 & 1.21 & 0.53 & 0.48 & 0.19 & 0.32 & $-0.40 \pm 1.37$ & $-0.53 \pm 1.29$ \\
Composite & - & 0.49 & 0.43 & 1.23 & 1.27 & 0.43 & 0.41 & 0.18 & 0.26 & $0.49 \pm 1.27$ & $0.43 \pm 1.32$ \\
\hline
\end{tabular}

For the HWB301 predictions, the composite condition is used at all three certification points. The statistics for 
the composite condition are computed by weighting the information equally between each of the individual sideline and cutback conditions. Incorporation of an approach condition is neglected as the vehicle noise is insensitive to the jet noise for that certification point. It is also important to recall that the Mach number for the sideline and cutback certification conditions is 0.25 while the experiments are at lower Mach numbers. It is also prudent to recall that the reference data prediction test method is technically only valid when the prediction matches the experimental conditions. The uncertainty for the jet from the composite condition is used despite this limitation, due to the absence of better information, as well as an indication from the results of Stone [19] showing that a similar jet had comparable levels of comparison error and uncertainty over the range of Mach numbers that are used in the HWB301 predictions here.

\section{Element Standard Uncertainties}

With an example of the jet noise prediction uncertainty process completed, the results of the other elements are aggregated to be used as inputs to the vehicle level analysis. Table 3 shows the standard uncertainties that were used previously, and updated values are given where the process has changed. The baseline values are used for an element if no updated information is available. The standard uncertainties have been updated for the jet, main and nose gear, and Krueger flap noise sources. It should be noted that although the process was updated for the nose gear uncertainty, the value did not change. For the Krueger flap, the improvements stem from an update to the bracket noise reflection modeling. The main gear uncertainty was reduced due to improvements in source modeling, and uncertainties due to the gear wake-flap interaction were excluded, since this effect is not present on an HWB configuration.

Table 3 Summary of Element Standard Uncertainties.

\begin{tabular}{lcccc}
\hline Element/Component & Type & Method & Baseline [3] & Current \\
\hline Fan & Source & Test & 2 & - \\
Jet & Source & Test & 3 & $0.43 \pm 1.32$ \\
Core & Source & Test & 3 & - \\
Main Gear & Source & Formulation & 1.4 & 1.1 \\
Nose Gear & Source & Formulation & 1.6 & 1.6 \\
Krueger Flap & Source & Formulation & 2.7 & 2.2 \\
Trailing Edge & Source & Test & 3 & - \\
Duct Liner & Noise Reduction & Inferred & 2 & - \\
Soft Vane & Noise Reduction & Inferred & 0.5 & - \\
Partial Landing Gear Fairing & Noise Reduction & Inferred & 0.25 & - \\
Fan and Core PAA & PAA & Test & 2 & - \\
Jet PAA & PAA & Test & 1 & - \\
\hline
\end{tabular}

Within this paper, there have been simplifications made regarding the uncertainty modeling, primarily driven by restrictions on the availability of quality validation data. One question that arises is whether more effort should be put into quantifying a single number for each of the uncertainties, or focusing on only some of the elements that contribute the most to the vehicle uncertainty and applying more detailed estimates for the uncertainty, for instance adding in angular or frequency dependence. For this reason, a separate Monte Carlo simulation is run, to determine the relative contribution of a subset of the sources which will have the most impact on the uncertainty in the cumulative certification level. This subset is limited to the Krueger flap, which is a highly ranked source for all three of the certification points, and the fan, which is a large contributor at cutback and sideline, as can be seen in Figure 9. The uncertainties of the fan PAA and the duct liner are also included. The soft vane uncertainty is neglected, as it has a relatively small uncertainty compared to the other fan effects. Depending on the relative contribution of the subset of elements to the overall uncertainty, it could be argued that effort is better spent refining the quantification of uncertainty for the element subset, or for the all the elements.

\section{Minimum Uncertainty}

A useful benchmark for better understanding the progress that has been made in reducing the uncertainty of a prediction is the establishment of a minimum uncertainty. In the previous study [3], this minimum value was estimated 


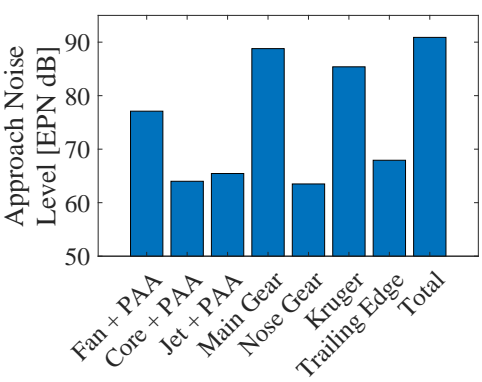

A

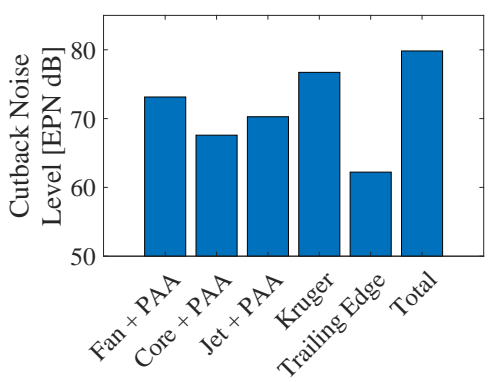

B

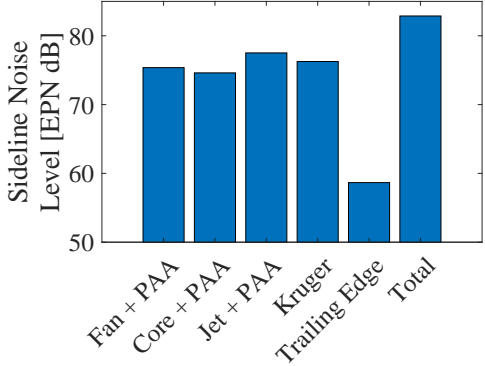

C

Fig. 9 The ranking of the sources is shown for each of the HWB301 certification point predictions. Approach, cutback, and sideline are shown in $\mathrm{A}, \mathrm{B}$, and $\mathrm{C}$, respectively.

from experience with the uncertainty in measuring a full scale vehicle in flight. It is important to recall that the scope of this study is to quantify the uncertainty that stems from the prediction models themselves. No uncertainties were considered with respect to the prediction inputs, nor were the modeling uncertainties considered for propagation of the sound from the source to the observer. For a vehicle in flight, the converse is true. The source models are dictated by the true physics, which are certain and unchanging. The uncertainty is due to changing inputs such as the flight path, engine power setting, or atmospheric properties. This is not to say that the minimum uncertainty defined from flight test experience has no value, but rather that it answers a separate question.

For the current question of estimating the minimum uncertainty of the models, a different approach is taken. From the details of the reference data prediction method, the minimum uncertainty for each element prediction is limited by the experimental uncertainty from which the comparison is drawn. The minimum uncertainty at the vehicle level is then determined by propagating the experimental uncertainties for each of the elements through a Monte Carlo simulation. Table 4 lists the uncertainties for each of the elements. Some modifications to the reported uncertainties were made in

Table 4 Summary of Minimum Standard Uncertainties.

\begin{tabular}{lcc}
\hline Element/Component & Value & Reference \\
\hline Fan & 0.24 & Envia et al. [30] \\
Jet & 0.25 & Bridges et al. [31] \\
Core & 0.64 & Hultgren et al. [32] \\
Main Gear & 0.28 & Guo et al. [21] \\
Nose Gear & 0.28 & Guo et al. [21] \\
Krueger Flap & 0.52 & Guo et al. [22] \\
Trailing Edge & 0.51 & SME \\
Duct Liner & 0.36 & Dahl et al. [33] \\
Soft Vane & 0.36 & Dahl et al. [33] \\
Partial Landing Gear Fairing & 0.25 & SME \\
Fan and Core PAA & 0.33 & Derived from fan \\
Jet PAA & 0.34 & Derived from jet \\
\hline
\end{tabular}

order to keep consistency with the approach used here. For the fan and jet, the references quantify the uncertainty by component for three different frequency ranges in decibels. The largest magnitude of uncertainty for each of the three frequency ranges quoted is used here as a conservative estimate. An additional difference is the fashion in which the component uncertainties are combined to quantify the overall uncertainty for the experiment. Within the references, the 
component uncertainties are combined using

$$
u_{E}=10 \log _{10}\left[1+\sum_{i}\left(10^{\left(\frac{u_{e, i}}{10}\right)}-1\right)\right]
$$

where the experimental uncertainties from different uncertainty sources, $u_{e, i}$, have units of $\mathrm{dB}$. This is a statement that the energy added by each uncertainty is uncorrelated to the overall level, but that the uncertainties themselves are perfectly correlated. For the component uncertainties stemming from those considered for the fan and jet (e.g. set point, pistonphone calibration, microphone distance to source) it is not expected that the underlying errors are correlated. Therefore, the experimental uncertainty is instead tabulated using a root sum square approach,

$$
u_{E}=10 \log _{10}\left[1+\sqrt{\sum_{i}\left(10^{\left(\frac{u_{e, i}}{10}\right)}-1\right)^{2}}\right]
$$

which states that the uncertainties are uncorrelated with the source level as well as each other. For the core, the quoted experimental uncertainty was assumed to be presented as $95 \%$ coverage interval. The same is true with the soft vane and duct liner, with the understanding that while these technologies were not the explicit subject of the reference, the same measurement technique would be used to determine the attenuation from them. The landing gear and Krueger flap uncertainties use the underlying data from the reference material as a starting point to determine an estimate of the repeatability. Other sources of uncertainties relating to the microphone calibration are then incorporated for consistency to the factors which were included in the other elements. When there was not a reference available, discussions with subject matter experts (SMEs) were used to give uncertainty estimates. The PAA effects, as well as the soft vane and MDOF duct liner, are implemented as suppressions, which is the difference between two test conditions. For this reason, the uncertainty is derived from the element uncertainty summed over two independent tests using Equation 14.

Another comparison that might be desired is to assess whether the uncertainty of the prediction is sufficient to meet the requirements for noise regulation. A prediction tool which is reliable enough to replace or significantly reduce flight testing is very attractive from a cost perspective. It should be made clear that there is no implication made here that ANOPP in its current state is a candidate for replacement of noise certification. However, the ultimate goal of aircraft noise prediction tools is to be able to reliably predict noise for a wide range of aircraft designs and operating procedures. It is for this reason that there is value in measuring progress using the uncertainty bounds that are allowed through certification. The uncertainty limits specified by regulation can be found in 14 CFR Part 36 A36.5.4.2, specifying that the $90 \%$ coverage limits must not exceed $\pm 1.5 \mathrm{EPNdB}$ for each of the three certification procedures. For consistency with the approach specified here, this corresponds to a $\pm 1.8 \mathrm{EPNdB} 95 \%$ coverage interval for each of the certification conditions, and a $\pm 3.1 \mathrm{EPNdB}$ for the cumulative certification limit.

\section{Results}

Three Monte Carlo simulations have been carried out at the vehicle level for the HWB301. The first is the current version of the vehicle uncertainty, which supersedes the results of the previous study [3]. The current results will be referred to as such in this section, while the results of the previous study are denoted as the baseline. The second is the rank prioritized vehicle uncertainty analysis, in which only the fan source, fan PAA, MDOF duct liner, and Krueger element uncertainties are propagated to the system level. Within the context of comparing the current case to the prioritized case, the current case is sometimes referred to as the complete case, as it includes uncertainties from every element. The final Monte Carlo simulation, referred to as the minimum uncertainty case, is the estimate of the minimum modeling uncertainty at the system level.

The previous sections have discussed the definition of the element uncertainties. Following their definition, elemental uncertainties are propagated to the vehicle level via direct Monte Carlo simulation. Blocks of 250 realizations are run until convergence is reached. The simulation is deemed to be converged when the variations in the running mean and standard deviation for all of the realizations within the most recent block are within the specified tolerance. For the vehicle statistics, the tolerance is \pm 0.005 , while for the element statistics, the tolerance is \pm 0.05 , ensuring that the vehicle and element results are converged to two and one decimal place(s), respectively.

The Monte Carlo simulation for the current case of the HWB301 uncertainty converged in 7250 realizations. Table 5 shows the relevant statistics for the vehicle uncertainty along with the deterministic prediction. Similar to the past results, the mean of the Monte Carlo simulations has a smaller margin than the deterministic prediction due to the 
application of one sided distributions at the element level. The offset between the deterministic prediction and the sample mean was $2.1 \mathrm{~dB}$ for the baseline results and $3.1 \mathrm{~dB}$ for the updated results here. The increase in the offset is largely due to the change to exponential distributions from one-sided Gaussian distributions, as the former has a larger mean bias from the deterministic prediction when both are prescribed the same distribution parameters. Similarly, use of exponential distributions rather than one-sided Gaussian distributions results in more positive skew and higher kurtosis at the element level, as is evident from Figure 6. With respect to the baseline results, the current results also show higher kurtosis. This originates with an additional change that was made at the element level. For elements that have been assigned a Gaussian distribution, the removal on the restriction to sampling of realizations within two standard deviations of the mean to unrestricted, simple sampling increases the kurtosis by allowing realizations from the tails of the distribution to be used in the Monte Carlo simulation.

Table 5 Sample statistics of the current Monte Carlo simulation.

\begin{tabular}{lccccc}
\hline Certification & Deterministic & Mean & Standard Deviation & Skewness & Kurtosis \\
\hline Approach & 90.89 & 91.41 & 1.03 & 0.14 & 3.09 \\
Cutback & 79.83 & 81.06 & 1.39 & 0.34 & 3.27 \\
Sideline & 82.88 & 84.24 & 1.31 & 0.39 & 3.47 \\
Cumulative & 253.60 & 256.71 & 2.16 & 0.14 & 3.04 \\
Margin & 40.40 & 37.30 & - & - & - \\
\hline
\end{tabular}

The probability densities of the samples for each of the certification points are shown in Figure 10, and are compared with a Gaussian PDF parameterized using the sample means and standard deviations. While they do tend to appear fairly Gaussian by eye, and have sample statistics of the skewness and kurtosis near that of a Gaussian population, chi-squared tests reject the the hypothesis that the samples come from Gaussian distributions for any of the certification points at the $5 \%$ significance level.

One additional item of interest is the reduction in the standard uncertainty relative to previous results. Here, the reduction from the 2016 analysis is roughly $10 \%$, while the past study [3] show that the 2016 results improved by about $20 \%$ over the 2013 results. A large part of the reason that the 2016 improvements were more effective is due to a focus on elements that had a larger impact on the total vehicle EPNL. Between 2013 and 2016, the fan noise prediction was improved by incorporation of a geared turbofan experimental database, while the landing gear and Krueger flap models were also updated. This observation is one of the motivating factors for the rank prioritized case. For the prioritized case, only the element uncertainties affecting the fan and Krueger noise levels are considered, as they are the highly ranked sources across all three certification points. For the Krueger, this is just the source level uncertainty while it includes the source level, PAA effects, and liner effects for the fan noise. However, the fan uncertainty for the prioritized case does not include the soft vane element uncertainty. The element level sample statistics between the prioritized and complete simulations for the fan differ by less than $2 \%$, indicating that neglect of the soft vane uncertainty is warranted. The simulation converged in 7750 realizations, and the sample statistics are given in Table 6.

Table 6 Sample statistics of the rank prioritized uncertainty Monte Carlo simulation.

\begin{tabular}{lccccc}
\hline Certification & Deterministic & Mean & Standard Deviation & Skewness & Kurtosis \\
\hline Approach & 90.89 & 91.17 & 0.72 & 0.77 & 3.96 \\
Cutback & 79.83 & 80.67 & 1.39 & 0.42 & 3.24 \\
Sideline & 82.88 & 83.45 & 0.85 & 0.85 & 4.06 \\
Cumulative & 253.60 & 255.30 & 1.79 & 0.34 & 3.14 \\
Margin & 40.40 & 38.71 & - & - & - \\
\hline
\end{tabular}

It can be seen that the sample means are closer to the deterministic values for the prioritized case than the complete case. This is simply because several of the elements are no longer random variables, and are fixed to the deterministic prediction. The cumulative standard deviation for the prioritized case is quite similar to the value for the complete case, differing by only $0.4 \mathrm{~dB}$. This corresponds approximately to a $90 \%$ ratio of the prioritized cumulative standard uncertainty to complete cumulative standard uncertainty in terms of pressure squared. With respect to the vehicle at 


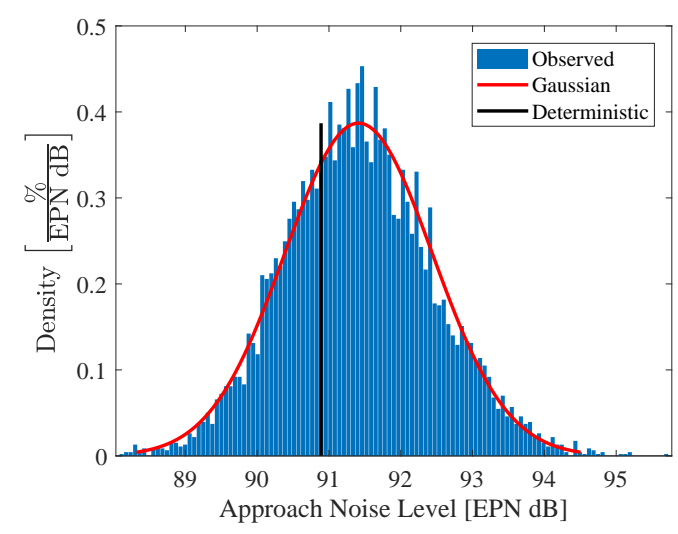

A

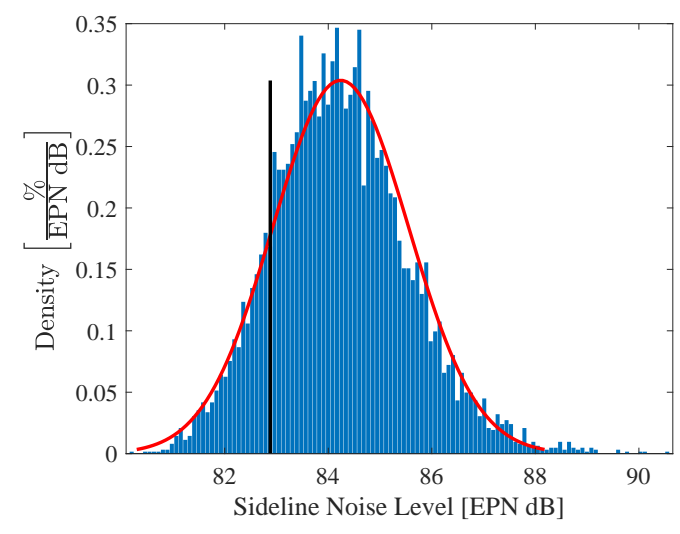

C

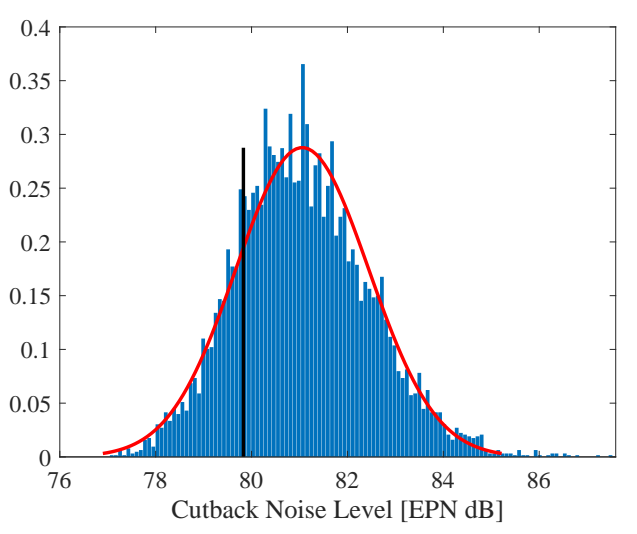

B

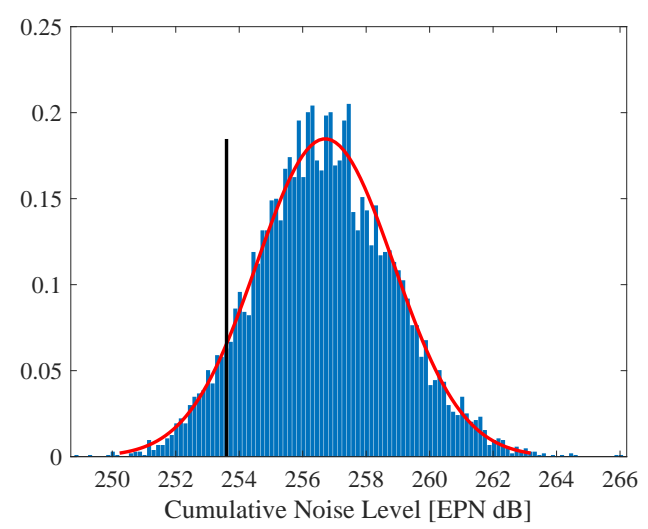

D

Fig. 10 Probability density plots are shown for approach, cutback, sideline, and cumulative distributions observed from the Monte Carlo simulation. A Gaussian PDF with the same mean and standard deviation as the sample is shown for reference, along with the deterministic prediction result.

hand, this implies that improved modeling of these two sources will result in the greatest impact on the cumulative uncertainty. As a more general point, it illustrates that a researcher need only focus on the uncertainty of the dominant elements to get a good first order uncertainty for a particular configuration. This is made abundantly clear when comparing the sample standard deviations for the cutback certification point between the prioritized and complete case. Recall from Figure 9 that for this certification point, the fan and Krueger flap are roughly equal at the element level and above any other source by $5 \mathrm{~dB}$, resulting in agreement in the standard deviation between the prioritized and complete simulations. This is not the case for the other two points where other elements have a considerable impact on the overall uncertainty, resulting in an incomplete capture of the vehicle uncertainty in the prioritized simulation.

For the prioritized case, the skewness is more positive than the corresponding values from the complete case. This is not due to the one-sided distributions, as this effect is seen on the approach certification point, where the Krueger flap is the dominant source of uncertainty. Recall that the distribution for the Krueger flap element is Gaussian. This effect comes from the nonlinear process of adding multiple element levels in decibels. For instance, at approach, addition of a larger deterministic value of the main gear level to the Gaussian Krueger flap distribution compresses the entire population, reducing the standard deviation at the combined level from its value at the element level. The upper tail tends to be stretched, while the lower tail of the distribution is compressed, positively skewing the distribution. When the deterministic value being added is larger than the mean of the distributed variable, the kurtosis will also increase. This effect explains why none of the vehicle level samples can be shown to come from a Gaussian distribution. The cumulative sample has statistics that are closer to a Gaussian distribution, as is expected from the central limit theorem.

With regards to the minimum uncertainty case, the trends in the statistics are similar to the other two cases. This case took only 5000 realizations to converge, likely due to the smaller magnitude of the elemental standard uncertainties 
relative to the convergence tolerance. The standard uncertainty for each of the certification points is approximately $0.3 \mathrm{~dB}$, resulting in cumulative standard uncertainty of $0.5 \mathrm{~dB}$. Recall that the purpose of quantifying the minimum uncertainty is to provide a target towards which to progress. However, while this does quantify the lower bound of the uncertainty that is possible when predicting the aircraft noise, it is not necessarily the best choice of a target minimum uncertainty. One challenge to using this minimum uncertainty as a target is that it assumes that prediction models which have been validated through subscale experiments are equally valid at full scale, essentially assuming that the models include all scale and integration effects. More achievable target uncertainties are the limits set by certification that were discussed in Section IV.D. The minimum uncertainty result here simply indicates that there is nothing inherent in the process that further restricts the uncertainty in the prediction.

Table 7 contains the probabilistically symmetric $95 \%$ coverage intervals for each of the Monte Carlo simulations, with reference to the margin to Stage 4 . This is chosen as a reference for consistency with NASA goal metrics, which were written prior to Stage 5 coming into effect. For conversion to a Stage 5 margin, the Stage 4 margins must be reduced by $7 \mathrm{EPNdB}$. The current results appear to be somewhat mixed, considering that the element standard uncertainties are less than or equal to their values in the previous study. However, recall that sampling at the element level is no longer restricted to be within two standard deviations of the mean, countering the effect of reducing the element standard uncertainties. It should be pointed out that despite this, the width of the $95 \%$ coverage interval has still been reduced by $1.1 \mathrm{EPNdB}$ in the current study.

There is still considerable progress to be made to decrease the size of the coverage interval toward either of the minimum values that have been proposed. The results of the minimum uncertainty Monte Carlo simulation, correspond to a $95 \%$ coverage interval of $\pm 1.0 \mathrm{EPNdB}$ for the cumulative level. Note that the coverage interval quoted within this paragraph is presented as symmetric only for an order of magnitude comparison with the other proposed minimums; Table 7 clearly shows that this range is skewed for the HWB prediction. This minimum uncertainty represents a case where there is perfect agreement between the prediction models and the experimental data to which they are compared, and under the assumption that there is no additional uncertainty from any scaling or integration effects. This minimum modeling uncertainty does not estimate the minimum uncertainties due to inputs or propagation modeling. A second minimum that has been proposed is the estimate of the uncertainty that one might expect in a flight test, with a coverage interval of $\pm 1.8 \mathrm{EPNdB}$ proposed for the cumulative level [3]. This is essentially a top-down version of the minimum uncertainty from the Monte Carlo simulation, where experimental uncertainty at the observer represents the lower bound. If the desire is to determine a minimum modeling uncertainty, the actual minimum modeling uncertainty is likely to be between the two. The controlled, repeatable environment of a wind tunnel can provide experimental data with lower uncertainty, while flight tests include scaling and integration effects which are not easily captured in subscale testing. Leveraging both types of tests appropriately will lead to robust models with reduced uncertainty.

While not an estimate of the minimum modeling uncertainty, a reasonable goal to set for reducing the modeling uncertainty is to use certification limits mentioned in Section IV.D, \pm 1.8 EPNdB for each certification point and $\pm 3.1 \mathrm{EPNdB}$ for the cumulative noise level. The closest the complete case results shown in Table 5 come to this goal occurs at the approach certification point. The approach result at first appears to be only $10 \%$ larger than the allowed uncertainty of $1.8 \mathrm{EPNdB}$ when the $95 \%$ coverage interval is expanded around the sample mean. When the coverage interval is expanded about the deterministic prediction, the uncertainty in the approach condition is roughly $40 \%$ larger than the allowed certification uncertainty. This increase is due to the inclusion of the bias between the deterministic prediction and mean of the Monte Carlo simulation. Despite the current state of the uncertainty for the complete case, the results of the minimum uncertainty case show that the prediction uncertainty can potentially be brought to a level where it is on par with the uncertainty required for flight test certification. The rank prioritized case results clearly show that this reduction cannot happen without improvement in the uncertainties affecting the fan and Krueger flap levels. For clarity, it should be understood that it is not being suggested that tools like ANOPP will ever be able to completely replace flight testing. Consistent with the approach to uncertainty adopted here, the tool can only be validated to the uncertainty of the experiment or flight test to which it is compared. Flight test certification will therefore always be the most accurate means of ascertaining the vehicle noise.

\section{Conclusions}

An updated approach for calculating the uncertainty of the predicted certification noise levels of an HWB301 has been presented. However, this approach is general enough to be applied to any aircraft concept. A detailed example of the reference data prediction test method has been carried out to estimate the uncertainty for the jet element. A process has been described for condensing the element uncertainty from a function of frequency and emission angle to a single 
Table 7 Summary of $95 \%$ coverage intervals for Stage 4 Margin.

\begin{tabular}{lcccc}
\hline Case & Deterministic & Lower Limit & Upper Limit & Span \\
\hline Baseline & 40.2 & 33.3 & 42.9 & 9.6 \\
Current & 40.4 & 32.9 & 41.4 & 8.5 \\
Rank Prioritized & 40.4 & 34.8 & 41.9 & 7.1 \\
Minimum Uncertainty & 40.4 & 38.7 & 40.6 & 1.9 \\
\hline
\end{tabular}

number representative of those conditions which contribute the most on an effective perceived noise level basis. For the jet element here, this process does not appear to be significantly different than the results of a simple averaging process. In general, this will depend on the quality of the model for the source within an element which has the highest impact on the EPNL relative to the overall performance of the element model.

The current Monte Carlo simulation for the HWB301 indicates that refinement in the quantification of the uncertainty, as well as improvements in the underlying source models themselves have led to a reduction of $1.1 \mathrm{EPNdB}$ in the $95 \%$ coverage interval relative to the previous study. The current results indicate that with $95 \%$ likelihood of coverage, the HWB301 concept at a Mid Term technology level has a margin of 33-41 dB. It is still important to note that the deterministic prediction is still the best prediction that can be made with the information at hand. The deterministic prediction is only 1.6 EPNdB below the upper end of the Mid Term range, so reaching the upper limit is plausible under certain circumstances. In order to increase the likelihood of this, more technologies would have to be included at the Mid Term level. This would only be possible if the technology readiness level of other technologies were increased to a point where they could logically be matured in the appropriate time frame. It is also important to note that the cumulative margin is highly skewed toward predictions which have a smaller margin than the deterministic prediction. This is driven by the usage of exponential distributions to predict the noise reduction technologies and PAA effects. As the reason for using the exponential distribution was to reflect the tendency for technologies to produce less noise reduction at full scale than subscale, this skewness could be reduced by incorporation of full scale data for PAA effects and noise reduction technologies. With full scale data, more confidence in the predictions of these elements would allow for the use of a more symmetric PDF, resulting in a more symmetric coverage interval around the deterministic prediction. Considering a situation where adding full scale data allows for a more symmetric PDF, the coverage interval would shift toward the upper limit of the Mid Term goal.

A rank prioritized Monte Carlo simulation which considered only the fan, engine acoustic liner, fan PAA, and Krueger uncertainties illustrated that a strong majority of the overall vehicle uncertainty can be attributed to the uncertainties of these elements. While the relative importance of these particular sources will vary based on their ranking for any given aircraft configuration, is is likely that they will make up a substantial contribution to any advanced configuration due to the continuing push towards increased BPR. As is well known, increasing BPR shifts the relative dominance of engine sources toward the fan. As the overall engine noise continues to decrease, airframe noise sources like the Krueger flap will also continue to become more important. This is particularly the case for configurations with engine noise shielding, a critical design feature for meeting the Mid Term and Far Term goals. As such, future modeling work should focus on a better understanding of this subset of aircraft noise elements. Validation of these improved source models will also require high fidelity experiments and flight data. This is perhaps most clear for the fan PAA effect. Recall that this element uses an exponential distribution due to a lack of extensive validation in flight, which contributes to the skewing of the coverage interval towards a lower Stage 4 margin. Increased confidence in the modeling of these effects at full scale will allow for use of a less conservative PDF for the fan PAA.

Apart from improving the fan, acoustic liner, PAA, and Krueger noise prediction methods, there are several areas that will benefit from continued work in the area of uncertainty quantification of unconventional aircraft configurations. While it has been shown with high confidence that the HWB301 can meet the Mid Term noise goal, the same question could be asked regarding the likelihood of the Far Term technology level HWB301 meeting its noise goal. Several technologies at the technology readiness level for this time frame have been shown to produce sizable reductions in the cumulative noise. For instance, the pod gear concept discussed in Thomas et al. [4] showed a 3.3 EPNdB reduction in cumulative noise. These concepts would benefit from a rigorous quantification of the uncertainty in the noise reduction, potentially lending more confidence in the ability of a concept to produce measurable benefit after maturation on a full scale vehicle. Finally, there is value in quantifying the uncertainty of other unconventional configurations. This will allow for comparison of the relative performance of different vehicles, where a vehicle with a lower deterministic 
margin but smaller coverage interval may outperform a configuration with a higher deterministic margin and larger coverage interval.

\section{Acknowledgments}

The Aircraft Noise Reduction Subproject of the Advanced Air Transport Technology Project is acknowledged for funding of this work. The authors would like to thank John Rawls and Stuart Pope for their support in the noise prediction calculations and Monte Carlo setup. Jason June would like to thank Ian Clark for many substantive conversations on the framing of the uncertainty process. The authors thank Eric Fay, Joshua Sams, and Jonathan Levy of the Advanced Concepts Laboratory, Analytical Mechanics Associates for the renderings of the HWB aircraft.

\section{References}

[1] National Aeronautics and Space Administration, "NASA Aeronautics: Strategic Implementation Plan,” NP-2017-01-2352-HQ, Washington, DC, 2017.

[2] Burley, C. L., Thomas, R. H., and Guo, Y., "Quantification of Acoustic Scattering Prediction Uncertainty for Aircraft System Noise Assessment,” AIAA Paper 2016-3041, May 2016. doi:10.2514/6.2016-3041.

[3] Thomas, R. H., Burley, C. L., and Guo, Y., "Progress of Aircraft System Noise Assessment with Uncertainty Quantification for the Environmentally Responsible Aviation Project," AIAA Paper 2016-3040, May 2016. doi:10.2514/6.2016-3040.

[4] Thomas, R. H., Guo, Y., Berton, J., and Fernandez, H., "Aircraft Noise Reduction Technology Roadmap Toward Achieving The NASA 2035 Goal,” AIAA Paper 2017-3193, June 2017. doi:10.2514/6.2017-3193.

[5] Lopes, L. V., and Burley, C. L., “ANOPP2 User’s Manual,” NASA TM-2016-21934, 2016.

[6] Bertsch, L., Dobrzynski, W., and Guérin, S., “Tool Development for Low-Noise Aircraft Design,” AIAA Paper 2008-2995, May 2008. doi:10.2514/1.43188.

[7] LeGriffon, I., "Aircraft noise modelling and assessment in the IESTA program with focus on engine noise," $22^{\text {nd }}$ International Congress on Sound and Vibration, 2015.

[8] Bertsch, L., Schäffer, B., and Guérin, S., "Towards an uncertainty analysis for parametric aircraft system noise prediction," $12^{\text {th }}$ ICBEN Congress on Noise as a Public Health Problem, 2017.

[9] Joint Committee for Guides in Metrology, "Evaluation of measurement data—Guide to the expression of uncertainty in measurement," Tech. Rep. 100:2008, 2008.

[10] Nickol, C. L., and Haller, W. J., "Assessment of the Performance Potential of Advanced Subsonic Transport Concepts for NASA’s Environmentally Responsible Aviation Project,” AIAA Paper 2016-1030, January 2016.

[11] Van Zante, D. E., and Suder, K. L., "Environmentally Responsible Aviation: Propulsion Research to Enable Fuel Burn, Noise and Emissions Reduction,” International Society for Air Breathing Engines, Paper 2015-20209, October 2015.

[12] Czech, M. J., Thomas, R. H., and Elkoby, R., "Propulsion airframe aeroacoustic integration effects for a hybrid wing body aircraft configuration," International Journal of Aeroacoustics, Vol. 11, No. 3, 2012, pp. 335-368.

[13] Hutcheson, F. V., Brooks, T. F., Burley, C. L., Bahr, C. J., Stead, D. J., and Stuart Pope, D., "Shielding of turbomachinery broadband noise by a hybrid wing body aircraft configuration," AIAA Paper 2014-2624, June 2014. doi:10.2514/6.2014-2624.

[14] Khorrami, M. R., Humphreys Jr., W. M., and Lockard, D. P., “An Assessment of Flap and Main Landing Gear Noise Abatement Concepts,” AIAA Paper 2015-2987, June 2015. doi:10.2514/6.2015-2987.

[15] Flamm, J. D., James, K. D., and Bonet, J. T., “Overview Of ERA Integrated Technology Demonstration (ITD) 51A Ultra-High Bypass (UHB) Integration for Hybrid Wing Body (HWB),” AIAA Paper 2016-0007, January 2016. doi:10.2514/6.2016-0007.

[16] Lytle, J. K., “The Numerical Propulsion System Simulation: An Overview,” NASA TM-2000-209915, June 2000.

[17] McCullers, L., “Aircraft Configuration Optimization Including Optimized Flight Profiles," Proceedings of the Symposium of Recent Experiences in Multidisciplinary Analysis and Optimization, NASA CP-2327, 1984, pp. 395-412.

[18] Ozoroski, T. A., "Description, Usage, and Validation of the MVL-15 Modified Vortex Lattice Analysis Capability," NASA CR-2015-218969, 2015. 
[19] Stone, J. R., Krejsa, E. A., Clark, B. J., and Berton, J. J., "Jet Noise Modeling for Suppressed and Unsuppressed Aircraft in Simulated Flight,” NASA TM-215524, April 2009.

[20] Bilwakesh, K. R., Emmerling, J. J., Kazin, S. B., Iatham, D., Matta, R. K., and Morozumi, H., "Core engine noise control program," FAA/RD-74/125, 1976.

[21] Guo, Y., Burley, C. L., and Thomas, R. H., "Landing Gear Noise Prediction and Analysis for Tube-And-Wing and Hybrid-WingBody Aircraft,” AIAA Paper 2016-1273, January 2016.

[22] Guo, Y., Burley, C. L., and Thomas, R. H., "Modeling and prediction of Krueger Device Noise,” AIAA Paper 2016-2957, May 2016.

[23] Fink, M. R., “Airframe Noise Prediction Method,” FAA-RD-77-29, 1977.

[24] Standard Values of Atmospheric Absorption as a Function of Temperature and Humidity, Society of Automotive Engineers, March 1975. Aerospace Recommended Practice 866, Revision A.

[25] Joint Committee for Guides in Metrology, "International vocabulary of metrology—Basic and general concepts and associated terms,” Tech. Rep. 200:2012, 2012.

[26] Joint Committee for Guides in Metrology, "Evaluation of measurement data-Supplement 1 to the "Guide to the expression of uncertainty in measurement"--Propagation of distributions using a Monte Carlo method," Tech. Rep. 101:2008, 2008.

[27] Coleman, H. W., and Steele, W. G., Experimentation, Validation, and Uncertainty Analysis for Engineers, $3^{\text {rd }}$ ed., John Wiley \& Sons, Hoboken, NJ, 2009.

[28] Viswanathan, K., “Quality of Jet Noise Data: Issues, Implications and Needs,” AIAA Paper 2002-0365, January 2002. doi:10.2514/6.2002-365.

[29] Viswanathan, K., "Does a Model-Scale Nozzle Emit the Same Jet Noise as a Jet Engine?" AIAA Journal, Vol. 46, No. 2, 2008, pp. 336-355. doi:10.2514/1.18019.

[30] Envia, E., Tweedt, D. L., Woodward, R. P., Elliott, D. M., Fite, B., Hughes, C. E., Podboy, G. G., and Sutliff, D. L., "Fan Noise Prediction," Assessment of NASA's Aircraft Noise Prediction Capability, edited by M. Dahl, 2012, NASA TP-2012-215653, ch. 5, p. 124.

[31] Bridges, J. E., Khavaran, A., and Hunter, C. A., “Jet Noise Prediction,” Assessment of NASA's Aircraft Noise Prediction Capability, edited by M. Dahl, 2012, NASA TP-2012-215653, ch. 8, p. 262.

[32] Hultgren, L. S., Miles, J. H., and Jorgenson, P. C., "Engine System and Core Noise," Assessment of NASA's Aircraft Noise Prediction Capability, edited by M. Dahl, 2012, NASA TP-2012-215653, ch. 3, p. 51.

[33] Dahl, M. D., Hixon, R., and Sutliff, D. L., "Further Development of Rotating Rake Mode Measurement Data Analysis," AIAA Paper 2013-2246, May 2013. 